\title{
CRITERION FOR HEAT TRANSFER MODELING OF UNSTEADY DROPLET COMBUSTION
}

\author{
Wen-Jei Yang \\ Department of Mechanical Engineering \\ University of Michigan \\ Ann Arbor, Michigan 48109
}

(Communicated by J.P. Hartnett and w.J. Minkowycz)

\section{ABSTRACT}

A criterion is developed which determines the unsteady temperature modeling of a single spherical fuel droplet burning in a nonconvective environment: the lumped (temporally varying but spatially uniform droplet temperature)-and distributed (temporally and spatially varying droplet temperature) -parameter models. It is concluded that for most industrial cases the distributed-parameter approach should be employed since the lumped-parameter one can not yield sufficiently accurate prediction.

\section{Introduction}

Experimental results [for example 1] have indicated that although the $\mathrm{d}^{2}$ law is fairly well obeyed throughout the combustion of a droplet, the transport processes of heat and mass are in a transient state during the major portion of the droplet lifetime. $\mathrm{d}$ is the droplet diameter. This is evidenced by small variations in the flame-front standoff ratio (the ratio of the flame front radius to the droplet radius) during the droplet burning and the changes in the temperature within the droplet. Theories of non-steady combustion are complex and still in their infancy.

Recent1y, two comprehensive surveys $[2,3]$ have been conducted on the literature pertinent to droplet and liquid combustion. Law et al [4] have presented a review of transient heating and liquid-phase diffusion in fuel droplet vaporization. One may summarize the unsteady heat transfer process as follows: There are three dominant modes of heat utilization at the droplet surface during the time history of droplet combustion. It begins with the mode of droplet heating which takes up roughly 10 to 20 percent of the 
droplet lifetime. During this period, significant variations in all of the combustion characteristics occur at rapid rates. The droplet surface layer becomes appreciably heated, whereas the surface regression rate and the flamefront standoff ratio can increase by an order of magnitude. Then, droplet evaporation becomes the dominant heat utilization mode. In a low pressure, constant environment, these two modes constitute the unsteadiness which is related to the heating of the droplet from its initial temperature to the quasi-steady value. Quasi-steadiness which corresponds to the final dominant heat utilization mode in droplet combustion is attained in about 20 to 30 percent of the droplet lifetime, in many cases. Under the assumption of spacially uniform and temporally constant droplet temperature, the quasisteady theory or the $\mathrm{d}^{2}-1$ aw model of Godsave [5] concludes that the droplet surface regression rate, the flame-front standoff ratio and the flame temperature remain constant over the entire droplet lifetime. Since overall quasi-steadiness can be achieved in about 20 to 30 percent of the droplet lifetime in numerous instances, the $\mathrm{d}^{2}-1$ aw has been extensively used either to provide rough estimates for the droplet evaporation rate or to serve as input to the formulation of more complex processes, for example spray analyses.

Heat transfer from the flame to the droplet core takes place in the following manner, see Fig. 1: First, the heat released by combustion flows through the fuel vapor space to the droplet surface in the form of radiation and conduction or convection. With part of the heat utilized for evaporation at the droplet surface, the rest then penetrates into the droplet by conduction alone in the absence of external convection or through combined action of conduction and convection when external convection is presented. While the droplet is still under heating, the surrounding gas phase has already achieved a quasi-steady state, since fuel drop has much higher heat capacity than its vapor [3]. Therefore, it is a common practice to consider that the gas phase is at quasi-steady state and the unsteady behavior of spherically, symmetric thin-flame combustion is solely controlled by the trans port mechanism within the droplet.

In order to understand the phenomenon of unsteady spherically symmetric droplet combustion, two models have been proposed which are applicable only in a motionless environment in the absence of natural or forced convection: lumped-parameter (or "rapid-mixing") and distributed-parameter (or "diffusionlimit") models. In the former model, the internal thermal resistance in the 
droplet is small compared with the surface resistance between the droplet and the environment that the droplet temperature may be considered spatially uniform and only temporally varying. On the other hand, the latter model deals with a system in which the internal and surface resistances are of comparable magnitude so that the droplet temperature must be treated as temporally as well as spatially varying with time. Since the theories are strictly for the noncirculating (no liquid motion) droplet, both terms "rapid-mixing" and "diffusion-1imit" do not make any sense physically.

Law and Sirignano [6] have employed the effective specific latent heat of vaporization $\mathrm{H}^{*}$ to describe the criteria for the onset or regime of the three dominant heat utilization modes: For each fuel droplet in combustion, the value of $\mathrm{H}^{*}$ decreases monotonically with time. Droplet heating is the dominant mode for $\mathrm{H}^{*}$ greater than 2 . In the range of $2>\mathrm{H}^{*}>1.1$, droplet evaporation dominates heat utilization. Overall quasi-steadiness can be considered to have been approached when $H^{*}$ decreases below 1.1. The $d^{2}$ law applies when $H^{*}$ is unity.

While the quantitative definition of the three dominant heat utilization modes marks a significant advance in understanding the heat transfer mechanisms of droplet combustion and evaporation, the criterion for the application of the two different theories, lump- and distributed-parameter approaches, has been left unspecified. In the present study, a criterion is developed which selects the heat transfer model for a fuel droplet in spherically symmetric combustion.

\section{Analysis}

Consider a liquid layer of thickness $2 \mathrm{~L}$ being exposed to a semi-infinite region of hot gas at temperature $T_{\infty}$. A quasi-steady gas phase is assumed [3]. Let the interfacial area and temperature be $A$ and $T_{S}$, respectively. Heat balance at the interface at any instant requires

$$
-k_{\ell} \partial T / \partial n=h\left(T_{\infty}-\dot{T}_{S}\right)
$$

wherein $\partial T / \partial n$ denotes the temperature gradient in the liquid phase; $k_{\ell}$, the liquid thermal conductivity; and $h$, the heat transfer coefficient including both the conductive and radiative components, $h_{c}$ and $h_{r}$, respectively. One may approximate the gradient as

$$
\partial \mathrm{T} / \partial \mathrm{n} \cong\left(\mathrm{T}_{\mathrm{c}}-\mathrm{T}_{\mathrm{s}}\right) / \mathrm{L}
$$


in which $T_{c}$ is the midplane temperature of the liquid layer. $T_{C}-T_{s}$ represents the maximum temperature difference in the system. The approximation becomes more realistic as the internal resistance of the system reduces. Equation (1) may be rewritten as

$$
\left(\mathrm{T}_{\mathrm{S}}-\mathrm{T}_{\mathrm{c}}\right) /\left(\mathrm{T}_{\infty}-\mathrm{T}_{\mathrm{S}}\right) \cong \mathrm{Bi}
$$

Here, $B i$ is the Biot number defined as $h L / k_{\ell}$, which signifies the ratio of the internal resistance $L /\left(k_{\ell} A\right)$ to the surface resistance $1 /(h A)$. When $B i$ is sufficiently small, the internal resistance becomes negligible compared with the surface resistance. Then, $\left(T_{S}-T_{C}\right)$ would be small enough so that $T_{S}$ may be considered the same as $\mathrm{T}_{\mathrm{C}}$. In other words, the 1 iquid phase has spatially uniform temperature. This concept may be generalized to a system of any geometry provided that $\mathrm{L}$ is the characteristic length of the system. It is well known in transient conductive heat transfer that in a system whose shape resembles a plate, a cylinder or a sphere, the error introduced by the assumption of spatially uniform temperature at any instant will be less than 5 percent when Bi is less than 0.1 [7]. Following this practice, the criterion for application of the lumped-parameter model is set as

$$
\mathrm{Bi} \leqq 0.1
$$

One should employ the distributed-parameter model when

$$
\text { B } \mathrm{i}>0.1
$$

When evaporation takes place at the liquid-gas interface, equation (1) should be modified as

$$
\mathrm{k}_{\ell}\left(\mathrm{T}_{\mathrm{S}}-\mathrm{T}_{\mathrm{c}}\right) / \mathrm{L}+\rho_{\ell} \lambda \dot{\mathrm{L}} \cong \mathrm{h}\left(\mathrm{T}_{\infty}-\mathrm{T}_{\mathrm{S}}\right)
$$

in which $\rho_{\ell}$ denotes the liquid density; $\dot{L}$, the speed of interfacial movement resulting from the phase change; and $\lambda$, the latent heat of evaporation. The equation may be rearranged to read

$$
\left(T_{S}-T_{C}\right) /\left(T_{\infty}-T_{S}\right) \cong B i-E
$$

where

$$
E=\rho_{\ell} L \dot{L} \lambda /\left[k_{\ell}\left(T_{\infty}-T_{s}\right)\right]
$$

The criterion for application of the lumped-parameter model is then modified as

$$
\mathrm{Bi}-\mathrm{E} \leqq 0.1
$$


The distributed-parameter model should be used if

$\mathrm{Bi}-\mathrm{E}>0.1$

For a liquid droplet of spherical form, $L=R_{1} / 3$ and $\dot{L}=\dot{R}_{1} / 3$, wherein $R_{1}$ and $\dot{R}_{1}$ are the radius and speed of $i$ ts change, respectively. In droplet combustion, $T_{\infty}$ and $T_{S}$ may be considered as the flame temperature $T_{f}$ and the saturation temperature of the 1 iquid phase at combustion pressure, respectively. The $d^{2}$ law gives $R \dot{R}=K / 8$ in which $K$ represents the burning coefficient or evaporation constant. Then, $E$ can be rewritten as

$$
E=\rho_{\ell} K \lambda /\left(72 k_{\ell} \Delta T\right)
$$

wherein $\Delta \mathrm{T}=\mathrm{T}_{\mathrm{f}^{-}} \mathrm{T}_{\mathrm{S}}$.

The heat transfer coefficient $h$ combines the effects of heat flow to the droplet by conduction and radiation and can be expressed as $h=h_{c}+h_{r}$. In the absence of convection, the heat transfer coefficient $h_{c}$ for the sphere is

$$
\mathrm{Nu}=\mathrm{h}_{\mathrm{c}} \mathrm{d} / \mathrm{k}_{\mathrm{g}}=2 \text {. }
$$

The radiative component $h_{r}$ can be evaluated using

$$
\mathrm{h}_{\mathrm{r}}=\sigma \alpha\left(\mathrm{T}_{\mathrm{f}}^{4}-\mathrm{T}_{\mathrm{s}}^{4}\right) / \Delta \mathrm{T}
$$

wherein $\sigma$ denotes the Stefan-Boltzmann constant and $\alpha$ is the absorptivity of the droplet surface. Hottel et al [8] have found that under industrial conditions $h_{\mathrm{r}} / h_{\mathrm{c}}$ can vary from roughly 0.02 to 0.2 and thus can be neglected in most applications.

\section{Results and Discussion}

From the definition of $\mathrm{B}_{i}$ and $\mathrm{Nu}$, one obtains the relationship

$$
B i=k_{g} N u / k_{\ell}
$$

or

$$
\mathrm{Bi}=2 \mathrm{k}_{\mathrm{g}} / \mathrm{k}_{\ell}
$$

in a nonconvective environment where $\mathrm{k}_{\mathrm{g}}$ is the gas thermal conductivity.

The quasi-steady theory yields the burning coefficient as

$$
\mathrm{K}=\left(8 \mathrm{k}_{\mathrm{g}} / \rho_{\ell} \mathrm{C}_{\mathrm{pg}}\right) \ln (1+\mathrm{B})
$$

in which $\mathrm{C}_{\mathrm{pg}}$ signifies the constant-pressure specific heat of the gas phase. $B$ is the transfer number defined as

$$
B=\left[C_{p g}\left(T_{\infty}-T_{s}\right)+Q_{n C}\right] / \lambda
$$


wherein $\mathrm{n}$ denotes the stochiometric fuel-oxidizer mass ratio; $\mathrm{Q}$, the heat of reaction per unit mass of fuel reacted; and $C_{\infty}$, the oxidizer mass fraction of the environment. In the pressure range of interest, the burning coefficient $K$ is experimentally determined to be of the order of $10^{-3} \mathrm{~cm}^{2} / \mathrm{sec}$ for combustion, which agrees well with equation (11).

Equations (7), (10) and (11) are combined to yield

$\mathrm{E} / \mathrm{Bi}=(1 / 18 \mathrm{~F}) \ln (1+1 / \mathrm{F}+J)$

in which $F=C_{p g}\left(T_{\infty}-T_{s}\right) / \lambda$ and $J=n Q C_{\infty} / \lambda$. Equation (13) is graphically illustrated in Fig. 2. For an octane droplet burning in the standard atmosphere at $300{ }^{\circ} \mathrm{C}, \mathrm{C}_{\infty}=0.232, \mathrm{n}=0.284$ and $\mathrm{T}_{\mathrm{f}} \cong 2200{ }^{\circ} \mathrm{K}$. Its physico-chemical propexties are $Q=10.7 \mathrm{Kcal} / \mathrm{gm}, \lambda=72.0 \mathrm{cal} / \mathrm{gm}, \rho_{\ell}=635 \mathrm{Kg} / \mathrm{m}^{3}, C_{\mathrm{pl}}=0.60 \mathrm{cal} / \mathrm{gm}-{ }^{\circ} \mathrm{K}$, $\mathrm{k}_{\mathrm{g}}=13 \mathrm{cal} / \mathrm{m}-\mathrm{h}-{ }^{\circ} \mathrm{K}, \mathrm{C}_{\mathrm{pg}}=0.35 \mathrm{cal} / \mathrm{gm}-{ }^{\circ} \mathrm{K}, \mathrm{T}_{\mathrm{S}}=399{ }^{\circ} \mathrm{K}$ and $\mathrm{k}_{\ell}=135 \mathrm{cal} / \mathrm{m}-\mathrm{h}-{ }^{\circ} \mathrm{K}$. One obtains $F=8.75, J=9.79$ and $E / B_{i}=0.0152$. Since $E$ is negligible compared with $B i$, equations (3) may be used as the criterion for heat transfer modeling instead of equations (6). However, equation (10) gives $\mathrm{Bi}=0.192>0.1$. This indicates that the lumped-parameter model will not accurately predict unsteady thermal behavior in the spherically symmetric combustion of an octane droplet and therefore the distributed-parameter approach should be adopted. For most industrial cases, the values of $F$ and $J$ range from 1 to 100 and 1 to 20, respectively. As shown in Fig. 2, the values of $E / B i$ in these ranges of $F$ and $J$ are low enough to justify the use of equations (3) as the criterion to determine the heat transfer model. However, since $\mathrm{k}_{\mathrm{g}} / \mathrm{k}_{\ell}$ is generally of the order of 0.1 to 0.3 , equation (10) gives the values of $B i$ between 0.2 and 0.6 . One may then conclude that for most industrial cases the lumped-parameter model cannot accurately predict unsteady thermal behavior of a fuel droplet in spherically symmetric combustion and therefore the distributed-parameter approach is the proper choice for more accurate theoretical prediction.

\section{Conclusion}

With a few exceptions, heat transfer theories of nonsteady combustion developed thus far are applicable to a single, spherical, noncirculating fuel droplet in a nonconvective environment. They can be classified into two categories: the lumped-and distributed-parameter models. Euqation (6) or (3) is the criterion to determine either of the two models should be selected for accurate prediction. It is concluded that for most industrial cases, the distributed-parameter approach should be employed since the lumped-parameter mode1 can not provide sufficiently accurate results on unsteady thermal be- 
havior in the combustion process. However, the conclusion does not apply to a droplet in a natural/forced convective environment for which an alternate criterion has to be developed.

\section{References}

1. H. Isoda and S. Kumagai, 7th Symp. (Int.) on Combustion, 523 (1958).

2. A. Williams, Combustion and Flame 21, I (1973).

3. G.M. Faeth, "Current Status of Droplet and Liquid Combustion," presented at the 1977 Spring Meeting of the Central States of Combustion Institute, Cleveland, Ohio (1977).

4. W.A. Sirignano and C.K. Law, "A Review of Transient Heating and LiquidPhase Mass Diffusion in Fuel Droplet Vaporization," Symp. on EvaporationCombustion of Fuel Droplets, Amer. Chem. Soc. (1976).

5. G.A.E. Godsave, 4th Symp. (Int.) on Combustion, 818 (1953).

6. C.K. Law and W.A. Sirignano, Combustion and Flame 28, 175 (1977).

7. F. Kreith, Principles of Heat Transfer, 3rd ed., p. 140, Intext Educational Publishers, New York (1973).

8. H.C. Hotte1, G.C. Williams and H.C. Simpson, 5th Symp. (Int.) on Combustion, 101 (1955).

9. A. Williams, Combustion of Sprays of Liquid Fuels, Chap. 3, Elek Science, London (1976). 


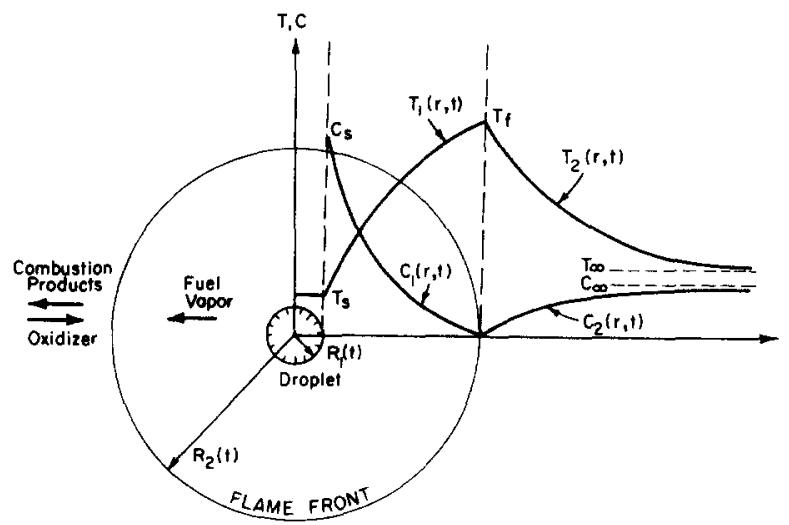

Fig. 1 Schematic diagram of buming fuel drop

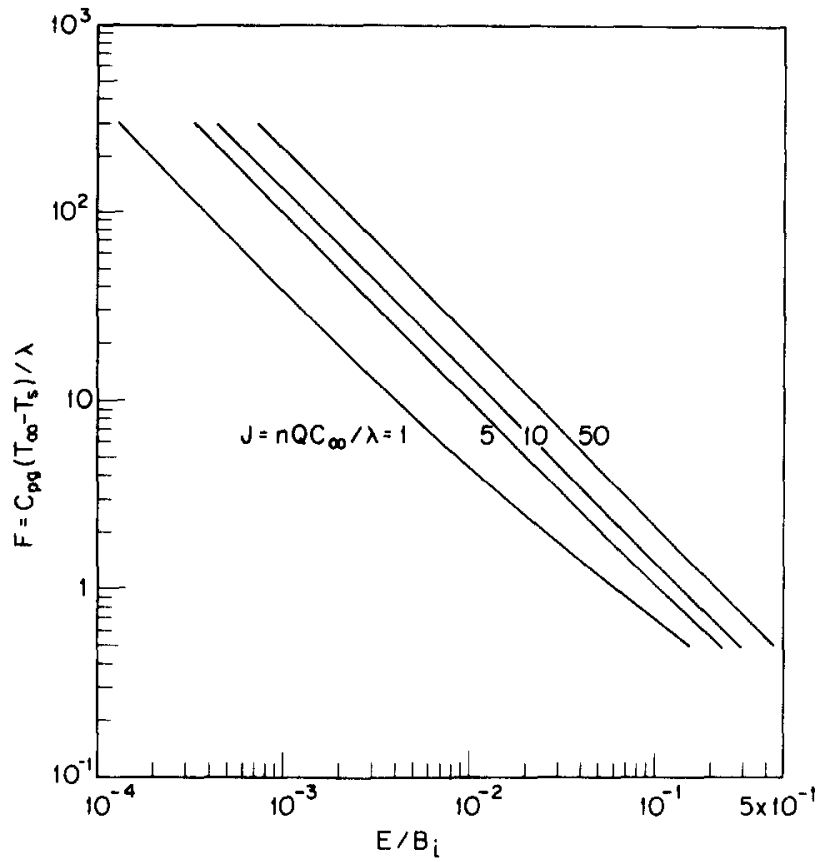

Fig. 2 Variations of $E / B_{i}$ with $F$ and $J$ 